Aim To evaluate the impact of this program in risk factors control and events.

Methods 514 consecutive MI patients hospitalised in six public hospitals. Data collection was obtained by a review of medical records and a 12-month interview (anthropometric and biochemical measurements, lifestyle information and pharmacological treatment). Predictors of good control were evaluated with multilevel analysis. Results follow-up was available in 398 patients (77.4\%), 75\% were male, aged 62.1 years $(\mathrm{SD} \pm 11.7)$. At the time of interview $8.6 \%$ were smokers; $24 \%$ reported regular physical activity; $78.6 \%$ were overweight or obese. The proportion of patients with raised systolic blood pressure was $46.3 \%$ and raised diastolic blood pressure was found in $35.4 \%$. 28.9\% had LDL cholesterol $\geq 100 \mathrm{mg} / \mathrm{dl}$ and $21.1 \%$ glucose $\geq 100 \mathrm{mg} / \mathrm{dl}$. In diabetic patients $(24.1 \%), 52 \%$ had glycosilated haemoglobin $\geq 7.0 \%$. The use of drug therapies at month 12 was: aspirin 95.5\%, $\beta$-blockers $70.6 \%$, ACE inhibitors $64.0 \%$ and statins $89.2 \%$. One year mortality was $6.8 \%$. Predictors for good control of risk factors were statin use (OR 2.64; CI 1.16 to 5.98) and control by cardiologist (OR 1.13; CI 1.01 to 1.27 ); diabetic patients have a poor control (OR 0.30; CI 0.15 to 0.61 ).

Conclusion Patients with MI have unhealthy lifestyles and a high proportion not achieved the goal for cholesterol and blood pressure management. A multidisciplinary approach is needed to improve secondary prevention in MI patients.

\section{P2-219 RACIAL/ETHNIC DISPARITIES IN THE TIMING OF DEATH DURING EARLY CHILDHOOD AMONG CHILDREN WITH CONGENITAL HEART DEFECTS}

doi:10.1136/jech.2011.142976j.52

\section{P2-221 THE EFFECT OF ENVIRONMENTAL EXPOSURE TO PHTHALATES ON TESTICULAR CARCINOGENESIS}

doi:10.1136/jech.2011.142976j.54

B Nguyen, ${ }^{*}$ J Gomes. University of Ottawa, Ottawa, Canada

Testicular germ cell tumour is the most common malignancy in young males and its incidence has been rising in recent years. Environmental and occupational exposures are believed to increase the risk of testicular cancer. The exposures along with genetic susceptibility may influence the risk of developing Testicular germ cell tumour even further. Phthalates are endocrine disruptors and are abundantly used as industrial plasticisers. Human exposure to phthalates occurs from the use of this substance in commerce. Di-2(ethylhexyl) phthalate (DEHP) is the most common phthalate found in consumer products. Post exposure DEHP is rapidly hydrolysed into its active form, mono-(2-ethylhexyl) phthalate (MEHP), a testicular toxicant and a carcinogen. The objective of this research was to determine the toxicity of MEHP. Possible mechanisms that may be involved in the pathogenesis of testicular atrophy from exposure to MEHP include FAS signalling, ROS signalling, NF$\mathrm{kB}, \mathrm{PPAR}$ and cAMP pathway. Alternative mechanisms may also be associated with the regulation of germ cell apoptosis leading to testicular atrophy. Relevant genes of interest that may be affected are Testisin, GSTP1, and MGMT. The expression of these genes was examined by RT-PCR in testicular germ cells exposed to MEHP in a dose- and time-dependent manner at concentrations of $1 \mu \mathrm{M}$, $10 \mu \mathrm{M}$, and $100 \mu \mathrm{M}$ at $24,48,72$ and $96 \mathrm{~h}$ time points. The findings of this study will allow for a better understanding of the role of phthalates in altering expressions in testicular germ cells and a better understanding of the process of testicular carcinogenesis.

\section{P2-222 RISK MARKERS FOR CORONARY HEART DISEASE AND TYPE 2 DIABETES IN CHILDHOOD: COMPARISON OF INDIAN CHILDREN LIVING IN INDIA AND THE UK}

doi:10.1136/jech.2011.142976j.55

Infants with congenital heart defects (CHD) have increased risk of childhood mortality; previous research indicates racial/ethnic differences in timing of death during infancy. However, less is known about racial/ethnic disparities in timing of death during early childhood. Texas Birth Defect Registry data were used in a retrospective cohort study of 19406 singleton, live-born infants, born with a CHD between 1 January 1996 and 31 December 2003 to non-Hispanic $(\mathrm{NH})$ white, NH-black, or Hispanic women. Registry data were linked to death records to ascertain deaths through 31 December 2005. Kaplan-Meier survival estimates were computed and HRs and $95 \%$ CIs were calculated from multivariable Cox-proportional hazard regression models to determine the adjusted effect of maternal race/ ethnicity on mortality for each specific CHD during the neonatal, post-neonatal and childhood periods. Racial/ethnic disparities in mortality were most pronounced during the post-neonatal period and persisted into early childhood. Among children who survived infancy, NH-Blacks with tetralogy of Fallot ( $\mathrm{HR}=3.61 ; 95 \%$ CI 1.25 to 10.47 ), coarctation of the aorta ( $\mathrm{HR}=3.13$; $95 \%$ CI 1.15 to 8.54 ) and ventricular septal defect ( $\mathrm{HR}=2.60 ; 95 \% \mathrm{CI} 1.31$ to 5.19$)$ were more likely to die in early childhood compared to similarly affected NH-Whites. No statistically significant differences in timing of death after infancy were found for Hispanics vs NH-Whites. Racial/ethnic disparities in timing of death in childhood for specific $\mathrm{CHD}$ diagnoses are present but of unknown aetiology. Elucidation of factors associated with early childhood CHD mortality will aid in development of public health and clinical strategies to reduce racial/ethnic disparities in childhood mortality.

\section{P2-220 WITHDRAWN}

${ }^{1} \mathrm{C}$ Nightingale, ${ }^{*} \mathrm{G}$ Krishnaveni, ${ }^{1} \mathrm{~A}$ Rudnicka, ${ }^{1} \mathrm{C}$ Owen, ${ }^{2} \mathrm{~S}$ Veena, ${ }^{3} \mathrm{~J}$ Hill, ${ }^{1} \mathrm{D}$ Cook, ${ }^{3} \mathrm{C}$ Fall, ${ }^{1} \mathrm{P}$ Whincup. ${ }^{1}$ St. George's, University of London, London, UK; ${ }^{2}$ Epidemiology Research Unit, CSI Holdsworth Memorial Hospital, Mysore, India; ${ }^{3}$ Medical Research Council Epidemiology Resource Centre, Southampton General Hospital, Southampton, UK

Introduction UK Indian adults have higher risks of coronary heart disease (CHD) and type 2 diabetes (T2D) than Indian and UK European adults. With growing evidence that $\mathrm{CHD}$ and $\mathrm{T} 2 \mathrm{D}$ risks begin before adulthood, we compared risk factor patterns in Indian children living in India and the UK.

Methods We compared markers of adiposity and cardiometabolic risk in 9-10 year-old Indian children in the Mysore Parthenon birth cohort study, India $(n=538)$ and in the cross-sectional Child Heart Health Study, England ( $\mathrm{n}=483$ ), which used comparable survey methods in 2007-2008 and 2004-2007 respectively. Small mean age and gender differences between studies were adjusted for in analyses. Results UK Indian children were taller and had markedly higher levels of BMI (mean difference $3.2 \mathrm{~kg} / \mathrm{m}^{2}$, \% difference $22 \%, 95 \% \mathrm{CI}$ 20 to $25 \%$ ) combined skinfold thickness (\% difference $36 \%, 95 \%$ CI 29 to $44 \%$ ), LDL-cholesterol (mean difference $0.4,95 \%$ CI 0.3 to $0.5 \mathrm{mmol} / \mathrm{l}$ ), systolic BP (mean difference 11.3 , 95\% CI 9.9 to $12.8 \mathrm{~mm} \mathrm{Hg}$ ) and fasting insulin (\% difference 141\%, 95\% CI 121 to $163 \%)$. These differences were similar in boys and girls; differences in LDL-cholesterol, blood pressure and insulin remained marked after adjustment for adiposity markers and pubertal status. Conclusions Substantial differences in cardiometabolic risk between UK Indian and Indian children are apparent before puberty. They do 